\title{
Diseño de un plan de acción para reducir la carga física biomecánica en empresas del sector del calzado del Valle del Cauca
}

\section{Design of a management plan to reduce the biomechanical workload in companies of footwear industry in Valle del Cauca}

\author{
Luis A. Saavedra-Robinson ${ }^{1}$, Valeria Marín-Londoño ${ }^{2}$, Camila Palacios-González ${ }^{3}$ \\ ${ }^{1}$ Departamento de Ingeniería Industrial, Pontificia Universidad Javeriana, Colombia. Email: 1.saavedra@javeriana.edu.co \\ ${ }^{2}$ Pontificia Universidad Javeriana, Colombia. Email: vvarela92@javerianacali.edu.co \\ ${ }^{3}$ Pontificia Universidad Javeriana, Colombia. Email: cpalacios11@ javerianacali.edu.co
}

Recibido: febrero 20, 2018. Aceptado: abril 25, 2018. Versión final: mayo 07, 2018.

\begin{abstract}
Resumen
Esta investigación presenta un abordaje sistémico al plan de mejoramiento de las condiciones ergonómicas en el sector del calzado. Se realizó un análisis jerárquico de procesos (AHP por sus siglas en inglés) incluyendo los métodos REBA y OCRA para dar priorizacion sobre los procesos que se van a intervenir. Se encontró que el mejor diseño organizativo para las empresas del sector es una estructura matricial de procesos; una distribución de planta lineal o en forma de "U" y que se debe atender criterios relacionados con la uniformidad de la iluminación dentro de las instalaciones. Finalmente, se rediseñó el puesto de trabajo a través de la metodología Design thinking, y se verificaron nuevamente los métodos REBA y OCRA, donde la valoración postural arrojó que en el caso de REBA, la valoración postural pasó de nivel de riesgo medio a bajo; y en el caso de la repetitividad (OCRA), pasó de no aceptable a muy leve.
\end{abstract}

Palabras clave: condiciones laborales; macroergonomía; planes de acción; sector del calzado.

\begin{abstract}
This research presents a systemic approach to the improvement of the conditions related to ergonomics in the footwear sector. We used a hierarchical analysis of processes, including biomechanical workload assessment (REBA and OCRA) to prioritize the productive processes that required intervention. Under the macroergonomic approach, it was found that the best organizational design for companies in the sector is a matrix structure of processes; a linear or "U" -shaped floor layout and, that criteria related to the uniformity of lighting within the installations must be met for work environment. Finally, the workplace was redesigned through the Design thinking methodology and the REBA and OCRA methods were verified again, where the postural assessment showed that in the case of REBA, the postural assessment went from medium risk level to low; and in the case of repetitiveness (OCRA), it went from not acceptable to very mild.
\end{abstract}

Keywords: working conditions; macroergonomics; management plans; footwear industry.

ISSN impreso: 1657 - 4583, ISSN en línea: 2145 - 8456

Este artículo puede compartirse bajo la licencia CC BY-ND 4.0 y se referencia usando el siguiente formato: L. Saavedra-Robinson, V. Marín, C. Palacios, "Diseño de un plan de acción para reducir la carga física biomecánica en empresas del sector del calzado del Valle del Cauca," Rev. UIS Ing., vol. 17, no. 2, pp. 241-252, 2018. Doi: https://doi.org/10.18273/revuin.v17n2-2018021 


\section{Introducción}

En el ámbito mundial, la ergonomía laboral es concebida generalmente por tres criterios. El primero, por una causa moral, donde se incluye esta disciplina como una acción protectora al trabajador; el segundo, por una obligación legal o reglamentaria, dada la vigente legislación en condiciones laborales, y el tercero, por una cuestión de estrategia y ventaja competitiva, donde se han evidenciado incrementos en la productividad, haciendo una mirada sistémica, integradora y global. Desde el punto de vista sectorial, en los últimos años se han realizado varios estudios ergonómicos en la industrial de calzado, principalmente en países como Brasil, España, Estados Unidos y Colombia; esto debido a que, a pesar de los avances tecnológicos que se han tenido en la manufactura en los últimos años, sus procesos son principalmente de carácter manual [1] y, por lo tanto, aumenta la probabilidad de que se desencadenen trastornos musculoesqueléticos en los trabajadores de este sector. Los autores Guimarães, Ribeiro, Renner y Oliveira realizaron una evaluación ergonómica en una de las plantas más grandes de calzado en Brasil, y, por medio del rediseño del sistema del trabajo, lograron incrementar la productividad y reducir el riesgo de desórdenes musculoesqueléticos [2].

Así mismo, el Instituto de Biomecánica de Valencia y Asepeyo desarrollaron estudios ergonómicos en España, donde se evaluaron puestos de trabajo en el sector del calzado en empresas representativas, en el que se plantearon propuestas de mejora en cuanto a posturas de trabajo, ritmo y pesos ejercidos [3]. En otros estudios, se han desarrollado análisis ergonómicos en la estación de trabajo de moldeado en una empresa de calzado, y se ha encontrado que se debía intervenir de manera urgente en factores de riesgos como posturas inadecuadas, movimientos repetitivos y levantamiento de fuerzas [4][5]. Las características de estos estudios convergen en que involucran aspectos de la ergonomía de manera desarticulada, sin una integración entre la micro y la macroergonomía.

Ahora bien, en Colombia, de acuerdo con Fasecolda, «las principales patologías laborales diagnosticadas corresponden a desórdenes musculoesqueléticos (85 $\%) »$. Adicionalmente, en la actualidad uno de los sectores en el país con mayor índice de trastornos musculoesqueléticos es el de manufactura [6], y según el Programa de Transformación Productiva del ministerio de comercio, industria y turismo, los departamentos representativos en la producción de calzado son Bogotá (35,96\% del total), Valle del Cauca (22,34\%), Antioquia $(21,65 \%)$, Cundinamarca $(6,46 \%)$, Caldas $(4,99 \%)$ y Santander $(2,63 \%)$ [7], lo que podría ser una ventaja competitiva para la región si mejoran las condiciones laborales presentes en el sector.

A partir de esta necesidad de aumentar la productividad y mejorar la economía del sector del calzado en el Valle del Cauca, surge una iniciativa de la Asociación Colombia de Industriales del Calzado, el Cuero y sus Manufacturas (Acicam) en realizar varios proyectos en las áreas de operaciones y ergonomía. De esta manera, este proyecto surge como parte del macroproyecto del área de Ergonomía [8], y tuvo como objeto desarrollar un plan de acción sistémico e integrado para los procesos productivos con base en la caracterización de la carga física biomecánica presente en el sector de calzado, y así proponer una metodología que permita abordar todos los ámbitos de la ergonomía, dado que en la literatura actual existe una carencia de planes de acción ergonómicos, pues solo se evidencian intervenciones que por lo general son de carácter correctivo.

Para los planes de acción se usa como base conceptual la microergonomía y la macroergonomía. La primera se centra en la interfaz hombre-máquina, mientras que la segunda se enfoca en el entorno y la organización [9]. Específicamente en este estudio, la macroergonomía se centrará en el diseño organizacional, distribución de planta (layout), diseño del proceso, entorno y capacitaciones; mientras que la microergonomía estará en función del diseño del puesto de trabajo y el diseño de la tarea. Este plan de acción se representará a través de un caso diseñado para una de las empresas participantes del estudio, a fin de ejemplificar las metodologías que abordaría el plan de acción.

\section{Metodología}

\subsection{Tipo de estudio}

Por las características del estudio, esta investigación tiene un enfoque mixto [10]. En primera instancia, se desarrollaron técnicas observacionales que permitieron establecer la línea base del plan de acción, incluyendo criterios subjetivos; sin embargo, su forma de evaluación y verificación optó por criterios cuantitativos que permitieron analizar los criterios para la elaboración de la propuesta de una manera sistemática y estandarizada. Por último, el estudio elabora un estudio de caso con una empresa piloto del sector para ejemplificar las características del plan de acción diseñado.

\subsection{Cálculo de la muestra}

Para realizar la muestra en el sector primero se identificó las empresas con las que se contaba Acicam en el Valle del Cauca, en total unas 74 empresas afiliadas. De estas, 42 se dedican a la producción de calzado de cuero. 
Finalmente 26 empresas decidieron participar inicialmente en el análisis. Luego, se estableció como criterio que las empresas deben tener los procesos principales para la producción de calzado: troquelado, desbaste, guarnecido, montaje, cardado y terminado. De esta manera, se escogen seis (6) empresas que contaban con la homogeneidad en sus procesos, y se decidió realizar el estudio con este tamaño muestral.

\subsection{Procedimiento}

Este proyecto, por ser la segunda fase del macroproyecto, cuenta con la identificación y la categorización de los riesgos por carga física biomecánica que provienen de la caracterización de la primera etapa. De esta manera, se deben determinar los procesos productivos más críticos en el sector, y para esto se utilizó la herramienta Analytic Hierarchy Process (AHP). Después de seleccionar los procesos productivos con mayor riesgo ergonómico, se desarrollaron planes para las tres etapas del plan de acción (corto, mediano y largo plazo).

El plan a corto plazo desarrolló el diseño del puesto de trabajo y el diseño de la tarea. Para ello, se utilizó la metodología design thinking [11], con el objeto de proponer de manera sistémica el rediseño del puesto de trabajo y la tarea del proceso crítico identificado. Para validar el plan de acción a corto plazo se aplican los métodos ergonómicos REBA, para evaluar la carga postural y OCRA, para valorar los movimientos repetitivos de los procesos involucrados.

Para el plan de acción a mediano plazo, se tuvieron en cuenta criterios del entorno (ruido, iluminación y estrés térmico), y para ello se utilizó el método de la constante de salón para hallar el número de puntos a medir en ruido e iluminación. Para el análisis del estrés térmico se utilizó el índice Welt bulb globe temperature (WBGT). Los resultados arrojados del entorno se compararon con los threshold limit value (TLV), los valores límites de referencia para el índice WGBT de la ISO 7243, la resolución del 83 y normas colombianas establecidas.

Ahora bien, en el diseño del proceso se evaluaron aspectos físicos, cognitivos y organizacionales. Respecto a los aspectos físicos, se usaron los datos encontrados en la primera fase del macroproyecto; en los cognitivos se utilizó el método NASA TLX para analizar la carga mental; y en los organizacionales se hace énfasis en la comunicación de los trabajadores distinguiendo la estructura organizacional.

Finalmente, para el plan de acción a largo plazo se consideró el diseño de planta, el diseño organizacional y las capacitaciones. Para el diseño de planta se desarrolló el método Systematic Layout Planning (SLP) que es un procedimiento con once pasos que permite identificar y evaluar las áreas involucradas; y el éxito de este método depende de las alternativas de solución que se propongan [12]. En el diseño organizacional se usó como base las tres eras clasificadas por Daft [13], en las que se establecieron las estructuras organizacionales de una empresa. Para terminar, las capacitaciones se desarrollan a largo plazo, porque es un tema que debe abordar la cultura del sector industrial y requiere un proceso detallado.

\section{Resultados}

\subsection{Definición de los procesos productivos}

Luego de aplicar el análisis jerárquico de procesos AHP, la técnica arrojó que los procesos con mayor riesgo por carga física biomecánica son guarnecido, $24 \%$; montaje, $21 \%$; terminado, $17 \%$; cardado, $15 \%$; desbaste, $14 \%$, y troquelado, $9 \%$. Es decir que el foco de atención de los planes de acción está sobre el proceso de guarnecido.

\subsection{Plan de acción a corto plazo}

Después de aplicar la metodología design thinking, el prototipo que se propuso para mejorar las condiciones laborales de los trabajadores del proceso de guarnecido abarca el diseño del puesto de trabajo, compuesto por una mesa y una silla ajustable para trabajar en posición semibípeda (sentado-parado), o sentado por completo; para la tarea se colocaron dos recipientes en los laterales de la mesa para que el proceso sea en la línea; y finalmente, la máquina tiene un botón para accionar la aguja en lugar del pedal. Además, el AHP que jerarquiza los elementos de este plan arrojó que se debe considerar primero el diseño del puesto de trabajo (63\%), después el diseño de la tarea $(26 \%)$ y luego la herramienta (11 $\%)$.

Primero, la silla está diseñada para que las personas puedan cambiar de posición (sedente o semibípeda), con el fin de que su trabajo sea más dinámico, se disminuya la fatiga $y$ se eviten enfermedades laborales. Las especificaciones antropométricas que debe tener la silla están entre el percentil 5 y 95 del sector, y son las siguientes:

- Ancho de la silla: corresponde al ancho de las caderas en posición sedente: $47,35 \pm 2,03 \mathrm{~cm}$.

- Profundidad o largo de la silla: es la distancia nalga poplítea en posición sedente. Será de 50,60 \4,4 cm.

- Espaldar de la silla: estará entre la altura hombros y la altura subescapular, ambas en posición sedente, entre: $76,75 \mathrm{~cm}$ y $63,47 \mathrm{~cm}$ respectivamente. Además, el ancho del espaldar tiene en cuenta el 
ancho de los hombros $(46,24 \pm 5,1 \mathrm{~cm})$.

- Altura ajustable: es la diferencia entre la altura cresta ilíaca en posición bípeda y la altura poplítea en posición sedente. Entonces, la altura ajustable de la silla oscila entre $6,94-13,50 \mathrm{~cm}$.

- El peso máximo que soportará es de $100 \mathrm{~kg}$.

A continuación, se ilustra el diseño del puesto de trabajo que simula el proceso de guarnecido con las especificaciones antropométricas de la silla y de la mesa
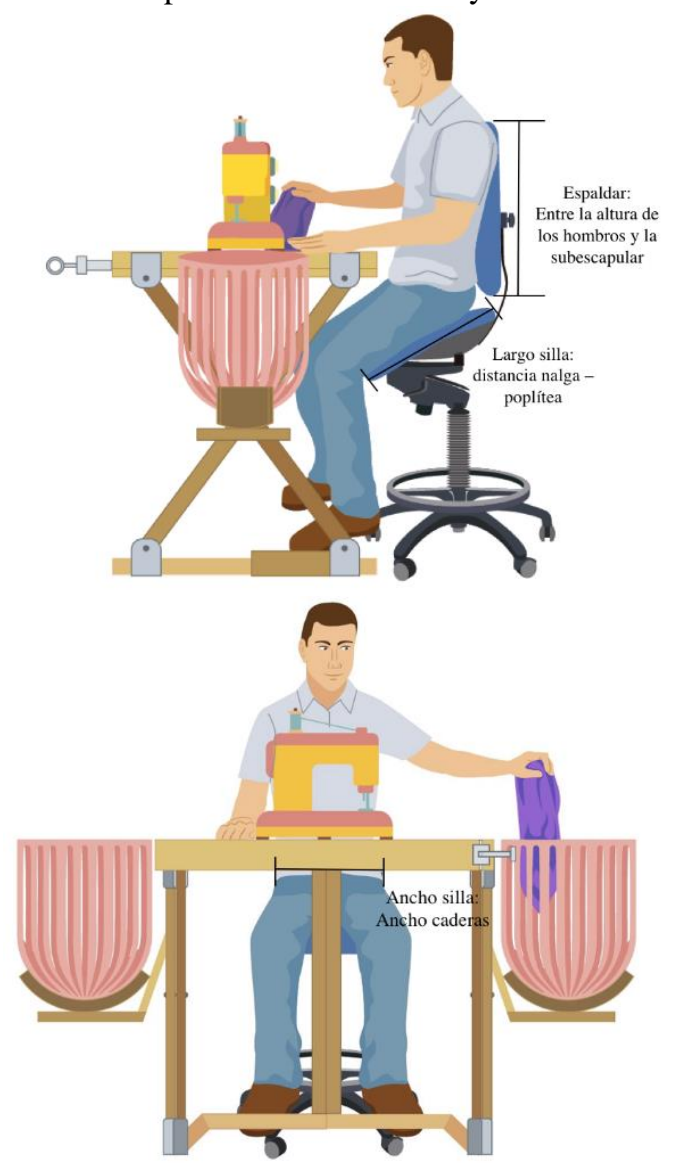

(véase figura 1). Asimismo, la mesa se adapta a la nueva posición del operario, por tanto, su altura es ajustable. Para esto, en sus laterales cuenta con dos soportes en forma de tijera que permiten el movimiento vertical por medio de un resorte que se acciona cuando se gira la manivela. La parte inferior de las tijeras está sobre unos rieles que guían el movimiento. El mecanismo que gradúa la mesa se encuentra ubicado únicamente en un lateral. Por tanto, esta mesa trabaja bajo un principio mecánico.

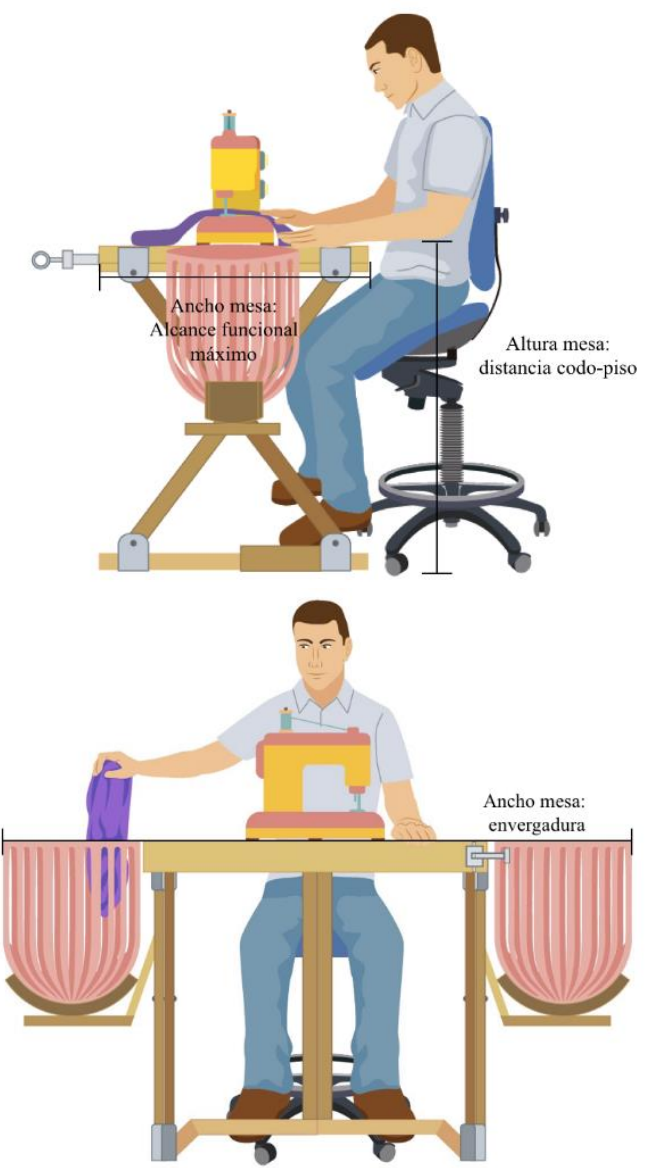

Figura 1. Puesto de trabajo - Guarnecido. Fuente: elaboración propia.

Dicho esto, las especificaciones antropométricas para la mesa están entre el percentil 5 y 95 del sector. Estas son:

- Ancho de la mesa: corresponde al alcance funcional máximo. Aproximadamente: $80 \pm 5 \mathrm{~cm}$.

- Largo de la mesa: es la envergadura. Será de $170 \mathrm{~cm}$.

- Altura ajustable: tiene en cuenta la altura del codo en posición sedente y en posición bípeda regulando la altura entre $70 \mathrm{~cm}$ y $110 \mathrm{~cm}$.

Para el diseño de la tarea se verificó que las secuencias de las actividades fueran ejecutadas en línea. En otras palabras, el operario con su mano derecha tomará el cuero del recipiente que se encuentra en el costado derecho de la mesa, luego realizara la guarnición de las partes del zapato en la máquina y después deposita con su mano izquierda el cuero guarnecido en el recipiente del costado izquierdo; esto con el fin de evitar las torsiones que se generaban al recoger la materia prima del suelo o dejar el producto guarnecido

Las especificaciones antropométricas para los recipientes laterales de la mesa: 
- Ancho de la canasta: al igual que el ancho de la mesa corresponde al alcance funcional, pero en este caso está entre el P5 y P75. Aproximadamente: $45 \mathrm{~cm} \pm$ $2,02 \mathrm{~cm}$.

- Largo de la canasta: se halla a través de un triángulo rectángulo con un ángulo de $30^{\circ}$, puesto que ese es el ángulo máximo de inclinación que una persona debe hacer y con un lado adyacente (ancho canasta) por medio de la ley del seno. Así pues, el largo de la canasta es $25,6 \mathrm{~cm}$, como se muestra en la siguiente figura (véase figura 2) y fórmula.

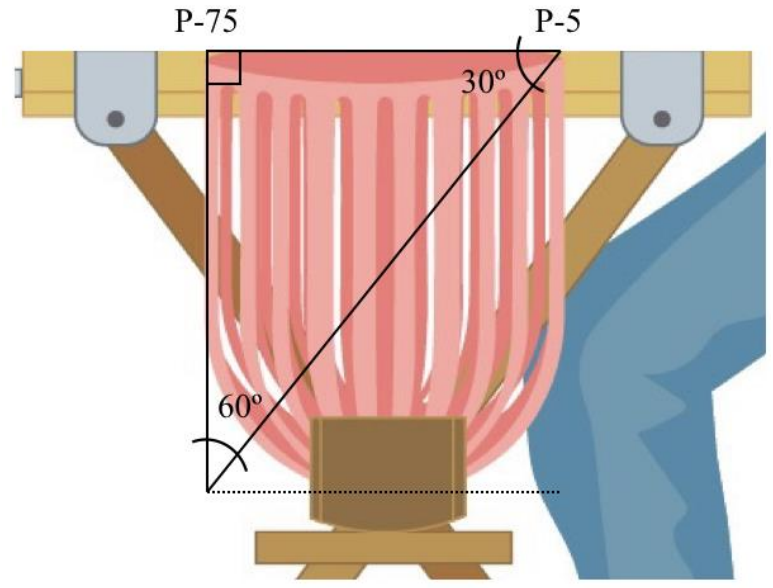

Figura 2. Medidas canasta. Fuente: elaboración propia.

$$
\begin{gathered}
\frac{45 \mathrm{~cm}}{\operatorname{Sen} 60^{\circ}}=\frac{b}{\operatorname{Sen} 30^{\circ}} \\
b=25,6 \mathrm{~cm}
\end{gathered}
$$

Tercero, la herramienta es la máquina de guarnecer, que es muy similar a una máquina de coser, y es accionada por un pedal. Sin embargo, con este diseño de la máquina se reemplaza el pedal por botones que permiten seleccionar la velocidad de la aguja y guarnecer (coser). Lo anterior con el fin de eliminar el movimiento repetitivo que generaba el pedal y reducir la fatiga.

Finalmente, se validó este plan por medio de los métodos REBA y OCRA para el prototipo del puesto de trabajo, y al compararlos con los resultados arrojados en la fase 1, se puede afirmar que el rediseño es efectivo. En REBA la puntuación pasó de nivel de riesgo medio (7) a bajo (3), y en OCRA pasó de no aceptable (promedio $=18,63$ ) a muy leve (promedio $=9,73$ ). En la siguiente tabla se expone la comparación de resultados en cada fase.
Tabla 1. Comparación de métodos ergonómicos para el rediseño.

\begin{tabular}{|c|c|c|c|c|}
\hline \multicolumn{4}{|c|}{ Análisis comparativo } \\
\hline & \multicolumn{2}{|c|}{ Resultado } & \multicolumn{2}{c|}{ Nivel de riesgo } \\
\hline Método & Fase 1 & Fase 2 & Fase 1 & Fase 2 \\
\hline REBA & 7 & 3 & Medio & Bajo \\
\hline OCRA & 18,63 & 9,73 & Medio & Muy leve \\
\hline
\end{tabular}

Fuente: elaboración propia.

\subsection{Plan de acción a mediano plazo}

En cuanto al entorno, después de hacer las mediciones de iluminación, ruido y estrés térmico se puede afirmar que existen hallazgos que deben ser tenidos en cuenta; por ejemplo, se realizó un análisis de los niveles de iluminación de cada uno de los procesos que son objeto de estudio de este proyecto. Para esto, se aplicó la metodología de la constante del salón descrita en los reglamentos técnicos colombianos referentes a la uniformidad del área de trabajo (véase tabla 2). A modo de ejemplo se aplicó la metodología a una de las empresas participantes, y estos fueron los resultados obtenidos:

Tabla 2. Mediciones para iluminación.

\begin{tabular}{|c|c|c|c|c|c|c|}
\hline \multirow{2}{*}{ Punto } & \multicolumn{3}{|c|}{ Medidas (Lux) } & \multirow{2}{*}{ Promedio } & FU=(Ei/Ep) & Uniformidad \\
\cline { 2 - 6 } & $\mathbf{1}$ & $\mathbf{2}$ & $\mathbf{3}$ & & & \\
\hline 1 & 210 & 183 & 194 & 195.7 & $32 \%$ & No \\
\hline 2 & 987 & 995 & 962 & 981.3 & $63 \%$ & No \\
\hline 3 & 526 & 528 & 527 & 527.0 & $85 \%$ & Sí \\
\hline 4 & 279 & 286 & 307 & 290.7 & $47 \%$ & No \\
\hline 5 & 522 & 499 & 491 & 504.0 & $82 \%$ & Sí \\
\hline 6 & 203 & 219 & 208 & 210.0 & $34 \%$ & No \\
\hline 7 & 748 & 776 & 786 & 770.0 & $80 \%$ & Sí \\
\hline 8 & 1040 & 1196 & 1155 & 1130.3 & $55 \%$ & No \\
\hline 9 & 889 & 990 & 944 & 941.0 & $66 \%$ & No \\
\hline
\end{tabular}

Fuente: elaboración propia.

En el caso piloto, no existe uniformidad en el recinto evaluado, dado que el porcentaje de uniformidad del área $(33 \%)$ se encuentra por debajo del porcentaje permitido en la normativa colombiana ( $75 \%)$

Con respecto al diseño del proceso, se tuvieron en cuenta las consideraciones cognitivas a través del método NASA TLX, que permite valorar la carga mental en un proceso (véase tabla 3). Este método se realizó en cada uno de los procesos de interés, y según la media ponderada global, el proceso con mayor carga mental es el montaje a continuación, donde se presentan los resultados del proceso de montaje. 
Tabla 3. Valoración del método NASA TLX para el proceso de montaje.

\begin{tabular}{|c|c|c|c|c|}
\hline \multicolumn{5}{|c|}{ Método NASA - TLX } \\
\hline Proceso: & \multicolumn{2}{c|}{ Montaje } \\
\hline Variable & Peso (Pi) & Puntuación & $\begin{array}{c}\text { Puntuación } \\
\text { convertida (Xi) }\end{array}$ & $\begin{array}{c}\text { Puntuación } \\
\text { ponderación }\end{array}$ \\
\hline Exigencias mentales (M) & 3 & 20 & 100 & 300 \\
\hline Exigencias fisicas (F) & 5 & 20 & 100 & 500 \\
\hline Exigencias temporales (T) & 4 & 20 & 100 & 400 \\
\hline Esfuerzo (E) & 2 & 17 & 85 & 170 \\
\hline Rendimiento (R) & 0 & 9 & 45 & 0 \\
\hline Frustación(Fr) & 1 & 10 & 50 & 50 \\
\hline Total & 15 & & & 1420 \\
\hline
\end{tabular}

Fuente: elaboración propia.
Así pues, la media ponderada global del proceso de montaje dio 94,67, a diferencia de las medias ponderadas globales de los otros procesos: troquelado, 73,33; desbaste, 64,33; guarnecido, 66,00; soladura, 55,33; y acabado, 85,67. Por consiguiente, el proceso de montaje se escoge para analizar el diseño de proceso de las consideraciones cognitivas. En el montaje se le da forma a la plantilla. Este proceso involucra varias máquinas como la de evaporación de agua, una saca - hormas y una conformadora (véase tabla 4). Las operaciones o actividades del montaje se muestran en la siguiente tabla, con el aspecto cognitivo ergonómico que predomina en cada una de estas.

Tabla 4. Aspectos cognitivos - montaje.

\begin{tabular}{|c|c|c|c|c|c|c|c|c|c|c|c|c|c|c|c|c|c|c|}
\hline \multirow{3}{*}{ 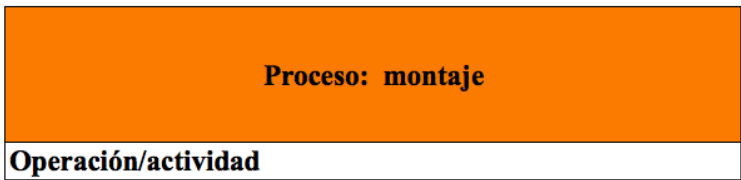 } & \multicolumn{18}{|c|}{ Tipo de consideración ergonómica (cognitiva) } \\
\hline & \multicolumn{3}{|c|}{$\begin{array}{c}\text { Exigencia } \\
\text { mental }\end{array}$} & \multicolumn{3}{|c|}{$\begin{array}{c}\text { Exigencia } \\
\text { física }\end{array}$} & \multicolumn{3}{|c|}{$\begin{array}{l}\text { Exigencias } \\
\text { temporales }\end{array}$} & \multicolumn{3}{|c|}{ Rendimiento } & \multicolumn{3}{|c|}{ Esfuerzo } & \multicolumn{3}{|c|}{ Frustración } \\
\hline & $\mathbf{A}$ & $\mathbf{M}$ & B & $\mathbf{A}$ & $\mathbf{M}$ & B & $\mathbf{A}$ & $\mathbf{M}$ & B & $\mathbf{A}$ & $\mathbf{M}$ & B & $\mathbf{A}$ & $\mathbf{M}$ & $\mathbf{B}$ & A & $\mathbf{M}$ & $\mathbf{B}$ \\
\hline 1. Colocar la plantilla en la parte inferior de la horma & $\mathrm{X}$ & & & & & & & & & & & & & & & & & \\
\hline 2. Ajustar la plantilla y la horma con taches & & & & $\mathrm{X}$ & & & $\mathrm{X}$ & & & & & & & & & & & \\
\hline 3. Pegar la capellada con engrudo & $\mathrm{X}$ & & & & & & & & & & & & & $\mathrm{X}$ & & & & \\
\hline 4. Ajustar la capellada con la conformadora de puntas y talones & & & & $\mathrm{X}$ & & & $\mathrm{X}$ & & & & & & & & & & & \\
\hline 5. Introducir el producto semielaborado en la máquina de calor & & & & & & & & & & & $\mathrm{X}$ & & & & & & & \\
\hline 6. Retirar los taches & & & & & & & & & & & & & & & & & $\mathrm{X}$ & \\
\hline
\end{tabular}

A= Alto; $M=$ Medio; $B=$ Bajo. Fuente: elaboración propia.

En los resultados que arroja el método NASA TLX por cada operación del proceso, se evidencia que este tiene exigencias mentales, físicas y temporales altas, y rendimiento, esfuerzo y frustración medias. Por tanto, se puede afirmar que el ritmo que tiene la tarea está directamente relacionado con el esfuerzo físico. Se sugiere entonces colocar la plantilla en la horma o pegar la capellada, y la horma y la capellada deben tener puntos guía para que el operario evite hacer cálculos.

\subsection{Plan de acción a largo plazo}

\subsubsection{Distribución de planta}

Para el diseño de planta a las empresas de calzado asociadas se les recomendó adoptar una redistribución similar a la arrojada por el SLP del caso aplicado (en forma de "U"), si solo cuentan con una entrada para hacer recepción y despacho, y si tienen dos entradas, se sugiere que adopten una distribución en línea. Sin embargo, para encontrar una propuesta de distribución que se adecuen a las características específicas de cada planta, las empresas deberían realizar los pasos del SLP.

Se tomó como piloto una de las participantes para aplicar el método SLP de distribución de planta. El primer paso fue determinar la relación entre las actividades y por ende su nivel de cercanía, actividades que tienen una interacción fuerte, su relación de cercanía debe ser proximal y así de manera sucesiva, como lo estipula la escala de valoración que proporciona el método (véase tabla 5). Para el ejemplo piloto, las relaciones de mayor importancia se dan entre las áreas de materia prima (MP), troquelado y desbaste; esto debido a la secuencia de trabajo. Ahora bien, el guarnecido y acabado de guarnecido, al igual que el premontaje y montaje son procesos que tienen una relación de proximidad, porque son procesos que realizan un trabajo similar.

Por otro lado, en la planta existen varias áreas donde la proximidad entre estas es insignificante. Por ejemplo, el área de soladura no debe estar cerca al área de troquelado ni a la bodega de materia prima, dado que en la secuencia de trabajo no están cercanos. 
Tabla 5. Tabla relacional de actividades.

\begin{tabular}{|c|c|c|c|c|c|c|c|c|c|c|c|c|c|c|}
\hline \multicolumn{15}{|c|}{ Tabla relacional de actividades } \\
\hline & $\begin{array}{c}\text { Recepción y } \\
\text { despacho }\end{array}$ & MP & Corte & Troquelado & Desbaste & Guarnecido & $\begin{array}{l}\text { Acabado de } \\
\text { guarnecido }\end{array}$ & Revisión & Premontaje & Montaje & Soladura & Acabado & Revisión & PT \\
\hline \multicolumn{15}{|l|}{ Recepción y despacho } \\
\hline MP & A & & & & & & & & & & & & & \\
\hline Corte & I & $\mathrm{A}$ & & & & & & & & & & & & \\
\hline Troquelado & $\mathrm{U}$ & $\mathrm{A}$ & A & & & & & & & & & & & \\
\hline Desbaste & $\mathrm{U}$ & $\mathrm{O}$ & $\mathrm{O}$ & A & & & & & & & & & & \\
\hline Guarnecido & $\mathrm{U}$ & $\mathrm{O}$ & $\mathrm{O}$ & $\mathrm{O}$ & A & & & & & & & & & \\
\hline Acabado de guarnecido & $\mathrm{U}$ & $\mathrm{O}$ & $\mathrm{O}$ & $\mathrm{O}$ & E & $\mathrm{A}$ & & & & & & & & \\
\hline Revisión & $\mathrm{U}$ & $\mathrm{U}$ & $\mathrm{U}$ & $\mathrm{U}$ & $\mathrm{U}$ & E & A & & & & & & & \\
\hline Premontaje & $\mathrm{U}$ & $\mathrm{U}$ & $\mathrm{U}$ & $\mathrm{U}$ & $\mathrm{U}$ & E & A & A & & & & & & \\
\hline Montaje & $\mathrm{U}$ & $\mathrm{U}$ & $\mathrm{U}$ & $\mathrm{U}$ & $\mathrm{U}$ & $\mathrm{O}$ & E & $\mathrm{U}$ & A & & & & & \\
\hline Soladura & $\mathrm{U}$ & $\mathrm{U}$ & $\mathrm{U}$ & $\mathrm{U}$ & $\mathrm{U}$ & $\mathrm{U}$ & $\mathrm{U}$ & $\mathrm{U}$ & $\mathrm{O}$ & A & & & & \\
\hline Acabado & $\mathrm{U}$ & $\mathrm{U}$ & $\mathrm{U}$ & $\mathrm{U}$ & $\mathrm{U}$ & $\mathrm{U}$ & $\mathrm{U}$ & $\mathrm{U}$ & $\mathrm{O}$ & $\mathrm{O}$ & A & & & \\
\hline Revisión & $\mathrm{U}$ & $\mathrm{U}$ & $\mathrm{U}$ & $\mathrm{U}$ & $\mathrm{U}$ & $\mathrm{U}$ & $\mathrm{U}$ & $\mathrm{U}$ & $\mathrm{U}$ & $\mathrm{U}$ & $\mathrm{U}$ & A & & \\
\hline PT & $E$ & $\mathrm{U}$ & $\mathrm{U}$ & $\mathrm{U}$ & $\mathrm{U}$ & $\mathrm{U}$ & $\mathrm{U}$ & $\mathrm{U}$ & $\mathrm{U}$ & $\mathrm{U}$ & $\mathrm{U}$ & $\mathrm{E}$ & A & \\
\hline
\end{tabular}

$\mathrm{A}=$ absolutamente necesario; $\mathrm{E}=$ especialmente necesario; $\mathrm{I}=$ importante; $\mathrm{O}=$ Proximidad ordinaria; $\mathrm{U}=$ insignificante.

Fuente: elaboración propia.

Del mismo modo, las áreas de premontaje y montaje no es necesario que vayan junto al área de producto terminado, es decir, no es indispensable que compartan el mismo espacio. Luego de haber determinado las relaciones, se procedió en llevar estas relaciones a la distribucion espacial de la planta piloto, representando el flujo de materiales y la relacion de proximidad. Para esto se usó la simbología de los procesos y la proximidad entre actividades proporcionadas por el método. A continuación, se muestra el diagrama para las áreas de la empresa de calzado a través del plano actual de la planta (véase figura 3 ).

Posteriormente se analizó el espacio requerido por cada actividad para realizar todas las operaciones necesarias. Para ello, se calculó el espacio estático (Ss), área que ocupa cada máquina; el espacio geométrico $(\mathrm{Sg})$, que refiere al espacio necesario para que se pueda acceder a las máquinas; y el espacio de evolución (Se), que está en función de una constante $\mathrm{K}$, que es la relación entre el promedio de la estatura de las personas que trabajan en el recinto y el promedio de las alturas de las máquinas del lugar. Así pues, las ecuaciones para determinar cada espacio son:

$$
\begin{aligned}
& S_{s}=\text { Ancho } \times \text { Largo } \\
& S_{g}=n \times S_{s} \\
& S_{e}=K \times\left(S_{s}+S_{g}\right)
\end{aligned}
$$

$K=\frac{\text { Promedio de la altura de las personas }}{2 \times \text { (promedio de las alturas de las máquinas })}$

Donde: $n=$ lados operables y $K=$ coeficiente de ocupación

Para este caso, la constante $\mathrm{K}$ se halló con la ecuación 8 , y para esta se usó el dato antropométrico de la altura de la población colombiana laboral, según el Instituto de seguros sociales (véase tabla 6).

Así, el espacio necesario total es de $221,50 \mathrm{~m}^{2}$ y el espacio requerido por cada proceso se ilustra a continuación (véase tabla 7). Una vez realizado el cálculo fue necesario contrastar lo requerido y el espacio real de la planta piloto. Específicamente, en la empresa de calzado se midieron las dimensiones de los espacios disponibles por cada proceso.

Tabla 6. Altura de la población colombiana laboral.

\begin{tabular}{|c|c|}
\hline Altura - dato antropométrico & h (m) \\
\hline Media masculino - Años: $20-59$ & 1.69 \\
\hline Media femenino - Años: $20-59$ & 1.56 \\
\hline Promedio & 1.62 \\
\hline
\end{tabular}

Fuente: Instituto de seguros sociales, 1995.

$$
K=\frac{1,62 \mathrm{~m}}{2 \times(1,47 \mathrm{~m})}=0.57
$$

Tabla 7. Área requerida vs. disponible por proceso.

\begin{tabular}{|c|c|}
\hline Proceso & $\begin{array}{c}\text { Área requerida } \\
\mathrm{m}^{2}\end{array}$ \\
\hline Recepción y despacho & 31.61 \\
\hline Materia prima & 33.28 \\
\hline Corte & 7.94 \\
\hline Troquelado & 5.58 \\
\hline Desbaste & 1.65 \\
\hline Guarnecido & 40.20 \\
\hline Acabado de guarnecido & 1.71 \\
\hline Premontaje & 4.30 \\
\hline Montaje & 99.14 \\
\hline Soladura & 11.25 \\
\hline Acabado & 2.40 \\
\hline Producto terminado & 11.91 \\
\hline
\end{tabular}

\begin{tabular}{|c|c|c|c|}
\hline \multirow{2}{*}{ Proceso } & Ancho & Largo & Área disponible \\
\cline { 2 - 4 } & $\mathrm{m}$ & $\mathrm{m}$ & $\mathrm{m}^{2}$ \\
\hline Recepción y despacho & 5.14 & 6.15 & 31.61 \\
\hline Materia prima & 15.43 & 6.00 & 92.58 \\
\hline Corte & 5.35 & 3.73 & 19.96 \\
\hline Troquelado & 8.69 & 2.37 & 20.60 \\
\hline Desbaste & 5.35 & 2.27 & 12.14 \\
\hline Guarnecido & 4.87 & 12.90 & 62.82 \\
\hline Acabado de guarnecido & 4.87 & 4.82 & 23.47 \\
\hline Premontaje & 3.82 & 6.23 & 23.80 \\
\hline Montaje & 5.14 & 13.18 & 67.75 \\
\hline Soladura & 5.14 & 6.39 & 32.84 \\
\hline Acabado & 5.14 & 4.43 & 22.77 \\
\hline Producto terminado & 8.88 & 2.27 & 20.16 \\
\hline
\end{tabular}

Fuente: elaboración propia. 
El área total disponible para los procesos de la empresa de calzado es $430,50 \mathrm{~m}^{2}$, sin embargo, el área total de la empresa es $575,54 \mathrm{~m}^{2}$; esto evidencia espacios subutilizados en la planta.

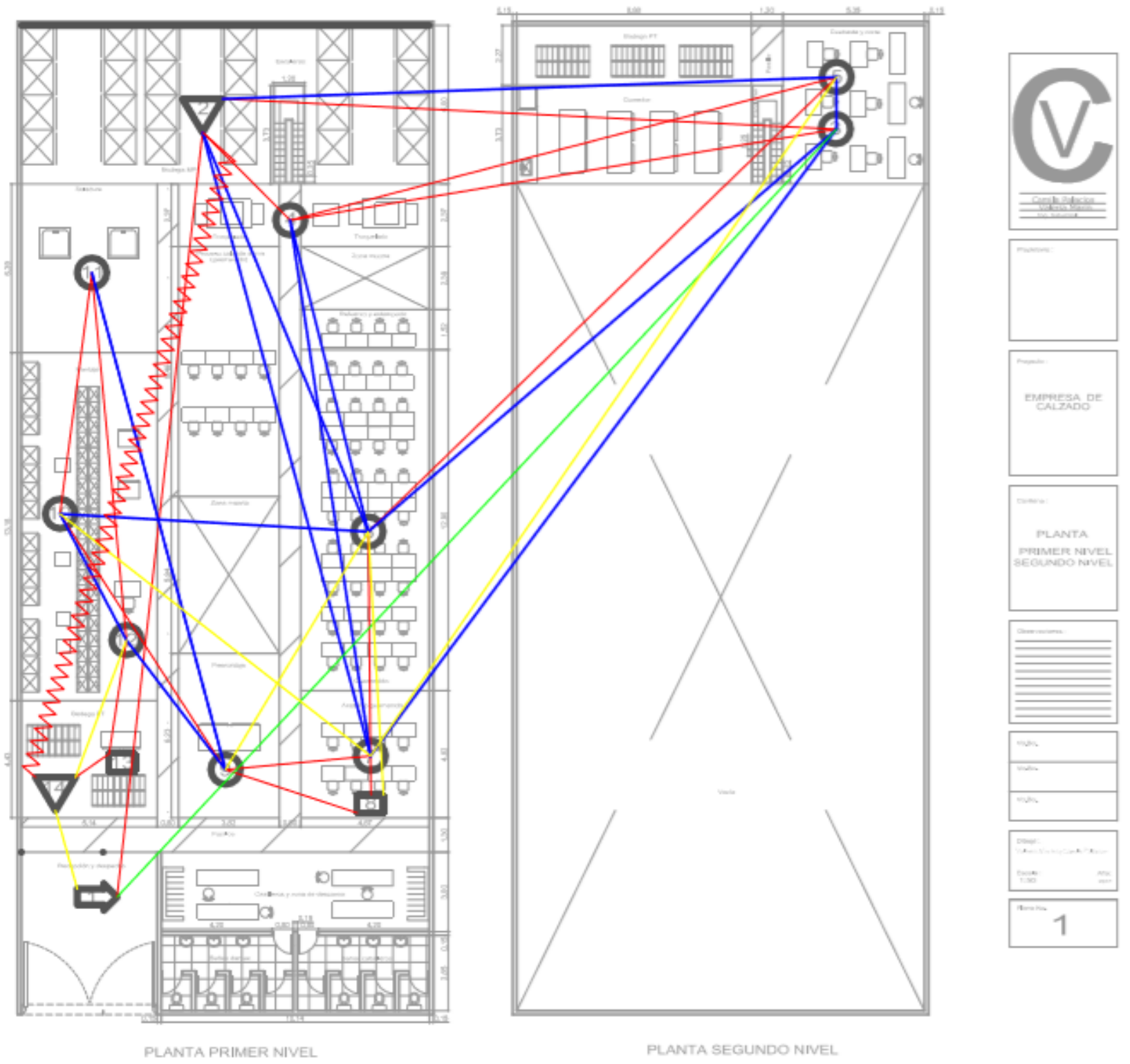

\begin{tabular}{|c|c|c|}
\hline Valor & Proximidad & Color \\
\hline A & Absolutamente necesaria & \\
\hline E & Especialmente importante & \\
\hline I & Importante & \\
\hline O & Ordinaria & \\
\hline U & Sin importancia & \\
\hline X & No deseable & \\
\hline XX & Altamente indeseabale & \\
\hline
\end{tabular}

Figura 3. Diagrama relacional de recorridos y actividades - situación actual. Fuente: elaboración propia.

\subsubsection{Desarrollo de soluciones}

Para desarrollar una solución de redistribución se tuvieron en cuenta tres aspectos: los elementos generales de distribución, principios básicos y la elaboración de la propuesta. Primero, los elementos generales se dividen en medios principales de producción como máquinas, operarios y materiales, y en medios auxiliares de 
producción como servicios sociales (comedores y baños) y generales (oficinas y almacenes). Segundo, los principios básicos de distribución son integración, mínima distancia recorrida, flujo de materiales, volumen ocupado, recursos humanos y flexibilidad - explicados anteriormente-. Tercero, en la elaboración de la propuesta se debe tener en cuenta toda la información recolectada en los pasos previos del SLP y el tipo de distribución más adecuada de acuerdo con el proceso.

En la figura 4 se ilustra la propuesta de redistribución para la empresa piloto. Para este desarrollo se tuvieron en cuenta los tres aspectos mencionados anteriormente; particularmente se debe resaltar que el tipo de distribución que se sugiere es en forma de "U", puesto que lo ideal es que el comienzo y el final del proceso se realice en el mismo espacio por el tipo de proceso y para disminuir el tiempo de transporte de materia prima y producto terminado. Asimismo, se tuvo un especial cuidado en el recorrido principal del producto, es decir, la relación de proximidad de un área con la otra para ubicarlas en el espacio. Es pertinente mencionar que como la planta contaba con espacion subutilizados y zonas de enseres en desuso, estos se reagruparon y se unieron todas las zonas de servicios sociales para la comodidad y bienestar de los trabajadores. Por tanto, es un tipo de distribución que cualquier planta de calzado que tenga los mismos procesos podría usar o adecuar.

\subsubsection{Evaluación y selección}

Para la evaluación y selección de las propuestas se recomienda usar el método carga-distancia. Este es un modelo matemático heurístico que se usa para evaluar las áreas de acuerdo con la proximidad de estas. Para esto, primero se debe hallar las coordenadas de cada una de las áreas en su centro estableciendo una cuadrícula en el plano de la planta. Luego, se hallan las distancias que hay entre cada área de acuerdo con el espacio requerido de estas (punto 5). Después, se establece el recorrido del producto por las diferentes áreas; se debe tener en cuenta el diagrama relacional del espacio del punto 7 . Finalmente, se suman las trayectorias que hace el producto y el total se multiplica por la cantidad de producto, a partir de esto se obtiene la carga-distancia de la distribución que se está evaluando. Por tanto, este procedimiento se hace por cada propuesta de distribución y el que tenga menor valor carga-distancia deberá ser la elegida.

Específicamente para este caso se usa el método de carga-distancia para evaluar la distribución actual en comparación con la redistribución que se propone, pues este es otro de los usos que se le puede dar al método: comprobar que la redistribución efectivamente es mejor a la distribución actual. A continuación, se muestran las distancias tanto de la situación actual como de la situación propuesta (véase tabla 8).

Tabla 8. Distancia entre áreas - plano actual y propuesta.

\begin{tabular}{|c|c|c|c|c|c|c|c|c|c|c|c|c|}
\hline \multicolumn{13}{|c|}{ Diagrama de relaciones con distancias - actual } \\
\hline & RyD & MP & $\mathrm{COR}$ & TRO & DES & GUA & ACG & PMON & MON & SOL & $\mathrm{AC}$ & PT \\
\hline RyD & 0.00 & & & & & & & & & & & \\
\hline MP & 31.72 & 0.00 & & & & & & & & & & \\
\hline COR & 35.56 & 7.42 & 0.00 & & & & & & & & & \\
\hline TRO & 26.00 & 7.23 & 9.62 & 0.00 & & & & & & & & \\
\hline DES & 35.56 & 7.42 & 0.00 & 9.62 & 0.00 & & & & & & & \\
\hline GUA & 16.79 & 18.89 & 20.30 & 11.77 & 20.30 & 0.00 & & & & & & \\
\hline ACG & 12.40 & 25.15 & 26.73 & 18.14 & 26.73 & 6.45 & 0.00 & & & & & \\
\hline PMON & 9.10 & 23.89 & 26.89 & 17.52 & 26.89 & 7.71 & 5.19 & 0.00 & & & & \\
\hline MON & 15.32 & 16.79 & 21.83 & 12.41 & 21.83 & 10.65 & 13.52 & 9.51 & 0.00 & & & \\
\hline SOL & 25.11 & 8.05 & 14.80 & 8.12 & 14.80 & 15.85 & 21.14 & 18.46 & 9.79 & 0.00 & & \\
\hline $\mathrm{AC}$ & 6.52 & 25.31 & 29.53 & 19.92 & 29.53 & 12.36 & 10.43 & 5.36 & 8.81 & 18.59 & 0.00 & \\
\hline PT & 6.52 & 25.31 & 29.53 & 19.92 & 29.53 & 12.36 & 10.43 & 5.36 & 8.81 & 18.59 & 0.00 & 0.00 \\
\hline \multicolumn{13}{|c|}{ Diagrama de relaciones con distancias - propuesta } \\
\hline & RyD & MP & COR & TRO & DES & GUA & ACG & PMON & MON & SOL & $\mathrm{AC}$ & PT \\
\hline RyD & 0.00 & & & & & & & & & & & \\
\hline MP & 9.63 & 0.00 & & & & & & & & & & \\
\hline COR & 10.10 & 5.03 & 0.00 & & & & & & & & & \\
\hline TRO & 10.61 & 6.23 & 1.20 & 0.00 & & & & & & & & \\
\hline DES & 12.71 & 7.59 & 2.96 & 2.11 & 0.00 & & & & & & & \\
\hline GUA & 13.46 & 10.93 & 5.90 & 4.70 & 3.91 & 0.00 & & & & & & \\
\hline ACG & 16.15 & 14.43 & 9.40 & 8.20 & 7.21 & 3.50 & 0.00 & & & & & \\
\hline PMON & 17.48 & 16.03 & 11.00 & 9.80 & 8.77 & 5.10 & 1.60 & 0.00 & & & & \\
\hline MON & 20.18 & 21.06 & 16.11 & 14.95 & 14.38 & 10.48 & 7.33 & 6.03 & 0.00 & & & \\
\hline SOL & 14.78 & 18.20 & 13.88 & 12.95 & 13.35 & 9.88 & 8.61 & 8.47 & 6.56 & 0.00 & & \\
\hline $\mathrm{AC}$ & 13.18 & 16.81 & 12.65 & 11.79 & 12.38 & 9.16 & 8.47 & 8.61 & 7.78 & 1.60 & 0.00 & \\
\hline PT & 7.90 & 12.56 & 9.41 & 8.95 & 10.35 & 8.65 & 9.99 & 10.92 & 12.44 & 6.90 & 5.30 & 0.00 \\
\hline
\end{tabular}

Fuente: elaboración propia. 


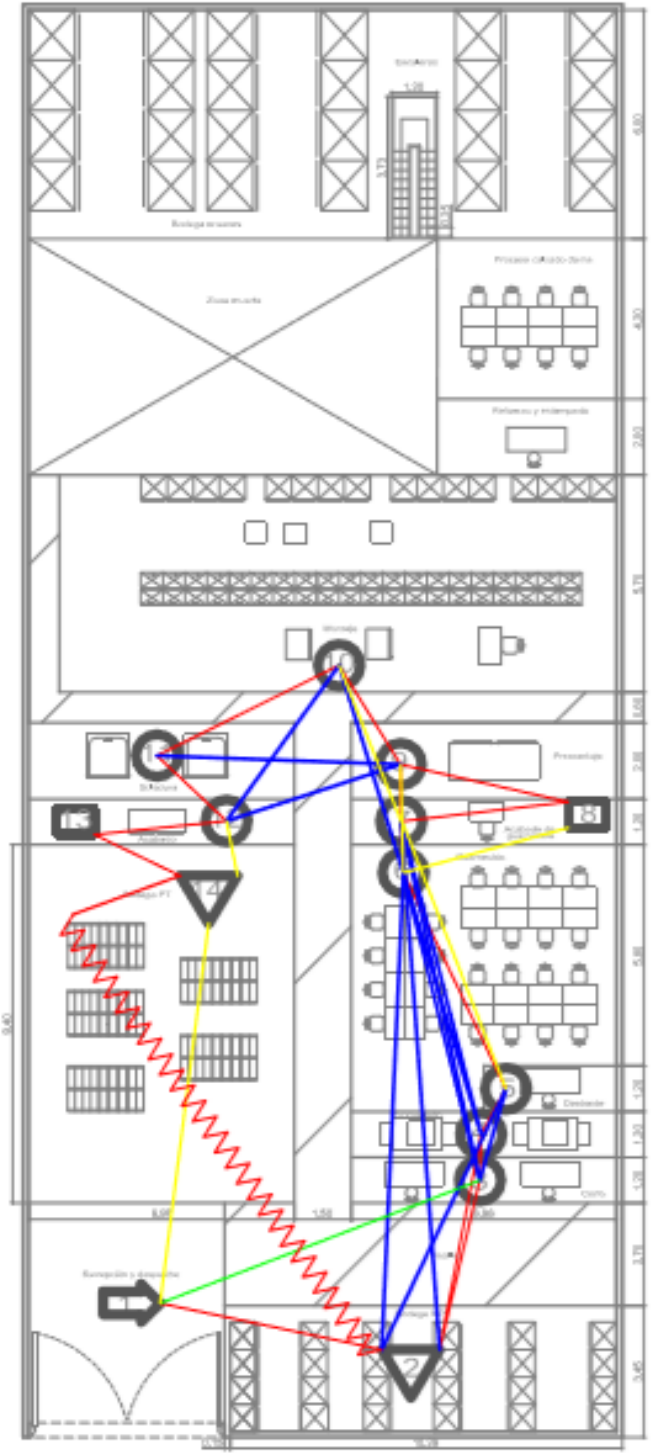

PLANTA PRIMER NIVEL

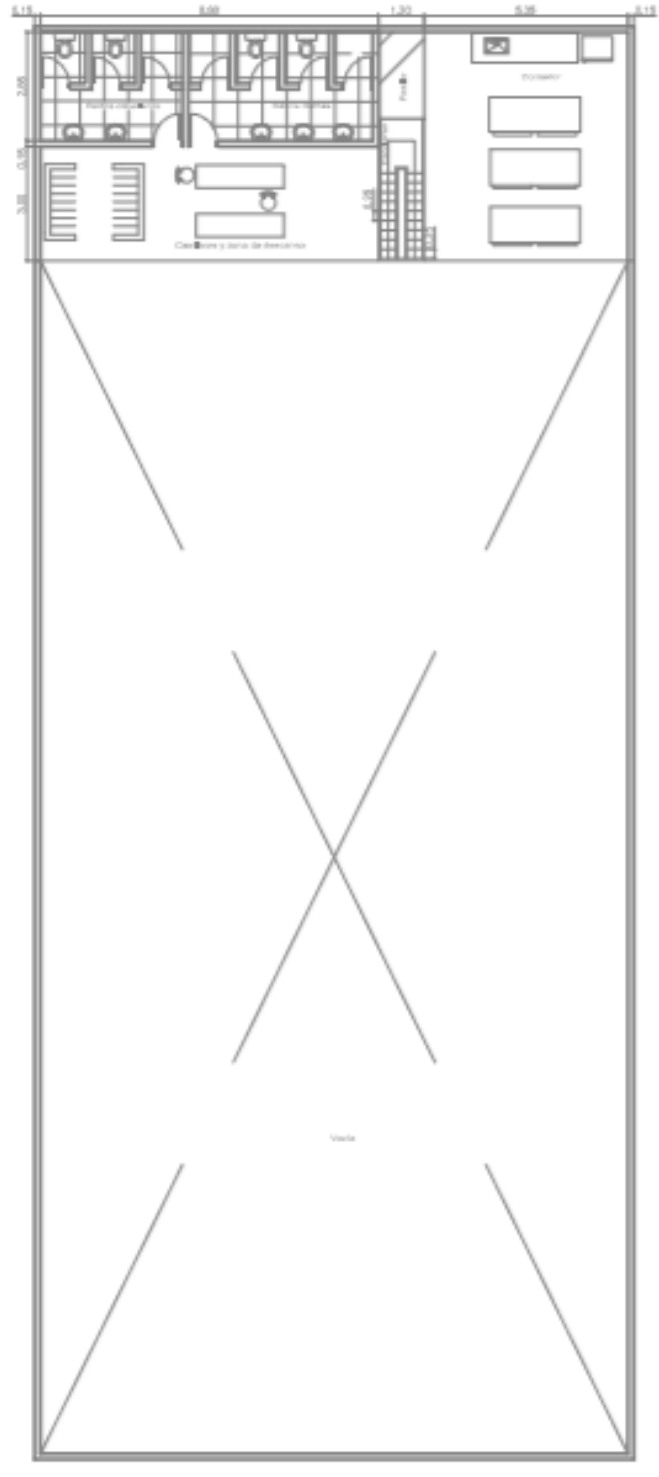

PLANTA SEGUNDO NIVEL

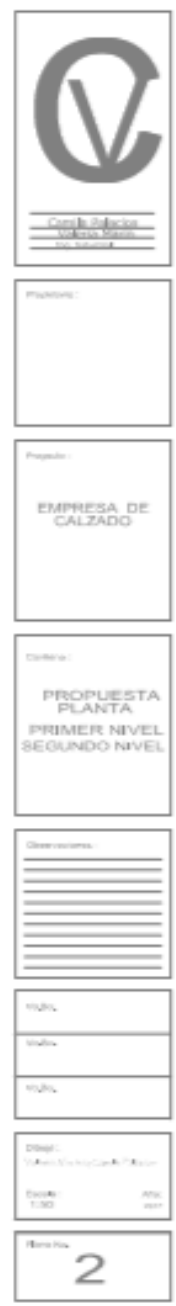

Figura 4. Plano planta de calzado - Propuesta. Fuente: elaboración propia.

De esta manera, se puede observar que después de evaluar la distribución actual y la redistribución, el método arroja que el valor de carga-distancia es menor para la redistribución $(11613,47)$ en comparación con la distribución actual $(32034,32)$ para corroborar la propuesta de redistribución.

\subsubsection{Diseño organizacional}

En el diseño organizacional, la estructura matricial y superposiciones horizontales se sugiere en este sector porque son empresas que están evolucionando de una jerarquía vertical a una estructura horizontal. Esta estructura explota las habilidades de los empleados al estar en su área e igualmente involucrados en un proyecto que puede estar conformado con personas de otros departamentos de la organización. También, esto permite que la comunicación sea más clara entre las áreas y los recursos sean compartidos. En síntesis, se llega a más metas en común, lo que genera mayor ganancia a la compañía. No obstante, el cambio de una estructura a otra no es fácil, y requiere de tiempo y trabajo en la cultura de la empresa; por tal razón este cambio se encuentra en el plan de acción a largo plazo. 
Para las capacitaciones se requiere tener en cuenta los siguientes aspectos:

- ¿Qué es la ergonomía?

- ¿Qué son las posturas forzadas? ¿Qué consecuencias conlleva una postura inadecuada?

- ¿Cómo evitar posturas inadecuadas en el proceso de montaje?

- Evaluación corta

Además, en los puestos de trabajo se recomienda pegar recordatorios visuales con el estándar de las posturas que deben realizar.

\section{Discusión}

Se ha realizado una comparación entre los estudios previos y los resultados del presente manuscrito, con el fin de evidenciar el alcance de las diferentes intervenciones en el sector del calzado (véase tabla 9).

Cabe resaltar que el presente estudio contiene los resultados del macroproyecto, incluida la caracterización de los riesgos por carga fìsica biomecánica y antropometría del sector calzado, del estudio realizado por Aragón y Ordoñez (2017).

Como se puede observar en la tabla anterior, el presente estudio abarca los diferentes enfoques de la ergonomía, integrándolos para generar planes de acción eficientes y contextualizados a las necesidades del sector. A pesar de que sus operaciones son de carácter manual, las tareas demandan otro tipo de exigencias: cognitivas y organizacionales. Por tal razón, el estudio fue basado en planes de acción que integran estos tres aspectos.

Por otro lado, dada la ausencia de planes de acción en ergonomía para el sector, se recurrió a indagar intervenciones en otros sectores para conocer metodologías y determinar qué herramientas de ingeniería industrial y diseño se podían incorporar para establecer nuevos criterios en los planes de acción en ergonomía. Por ejemplo, Analytic Hierarchy Process (AHP), Design thinking, Systematic Layout Planning (SLP) y demás presentados en este artículo.

Tabla 9. Comparación entre presente estudio y previos.

\begin{tabular}{|l|c|c|c|c|c|c|c|c|c|}
\hline Ergonomía & $\begin{array}{c}\text { IBV \& } \\
\text { Asepeyo } \\
\text { Autores }\end{array}$ & $\begin{array}{c}\text { Gomes } \\
\text { et al. } \\
{[2012]}\end{array}$ & $\begin{array}{c}\text { Baxley \& } \\
\text { Kuhens } \\
{[2013]}\end{array}$ & $\begin{array}{c}\text { Callegaro } \\
\text { et al. } \\
{[2013]}\end{array}$ & $\begin{array}{c}\text { Muñoz \& } \\
\text { Restrepo } \\
{[2014]}\end{array}$ & $\begin{array}{c}\text { Guimarães } \\
\text { et al. } \\
{[2014]}\end{array}$ & $\begin{array}{c}\text { Costa } \\
\text { et. al } \\
{[2015]}\end{array}$ & $\begin{array}{c}\text { Presente } \\
\text { Estudio } \\
{[2017]}\end{array}$ \\
\hline Física \\
\hline Antropometría & $\mathrm{X}$ & $\mathrm{X}$ & & & & & & $\mathrm{X}$ \\
\hline Carga postural & $\mathrm{X}$ & $\mathrm{X}$ & $\mathrm{X}$ & & $\mathrm{X}$ & & $\mathrm{X}$ & $\mathrm{X}$ \\
\hline Repetitividad & $\mathrm{X}$ & $\mathrm{X}$ & $\mathrm{X}$ & & $\mathrm{X}$ & & & $\mathrm{X}$ \\
\hline Cognitiva & & & & & & $\mathrm{X}$ \\
\hline Carga mental & & & & & & & \\
\hline Organizacional & & & & & & & & $\mathrm{X}$ \\
\hline Socio-técnico & & & & & & & $\mathrm{X}$ \\
\hline Diseño Org & & & $\mathrm{X}$ & & & & $\mathrm{X}$ \\
\hline Autoreporte & & &
\end{tabular}

IBV: Instituto biomecánico de Valencia. Fuente: elaboración propia.

Tabla 10. Acciones a corto plazo relacionadas con las intervenciones ergonómicas.

\begin{tabular}{|c|c|c|}
\hline Autor & Sector & Acción a corto plazo \\
\hline $\begin{array}{c}\text { Rodríguez \& } \\
\text { Pérez [s.f] }\end{array}$ & Imprenta & $\begin{array}{c}\text { Diseño de herramienta, } \\
\text { establecimiento de rotaciones y } \\
\text { nuevo método de trabajo. }\end{array}$ \\
\hline $\begin{array}{c}\text { García et al. } \\
\text { [2012] }\end{array}$ & Químico & $\begin{array}{c}\text { Adecuaciones del puesto de } \\
\text { trabajo. }\end{array}$ \\
\hline $\begin{array}{c}\text { SGS Tecnos } \\
\text { [2008] }\end{array}$ & Alimentos & $\begin{array}{c}\text { Modificaciones de la tarea, el } \\
\text { puesto de trabajo y la } \\
\text { herramienta. }\end{array}$ \\
\hline $\begin{array}{c}\text { Fund. Prev. } \\
\text { RL [s.f] }\end{array}$ & Construcción & $\begin{array}{c}\text { Establecimiento de pausas y } \\
\text { rotaciones, adecuaciones en el } \\
\text { puesto de trabajo. }\end{array}$ \\
\hline $\begin{array}{c}\text { IBV \& Fund. } \\
\text { Prev. RL } \\
\text { [2011] }\end{array}$ & Construcción & $\begin{array}{c}\text { Planificación de las tareas, orden } \\
\text { y aseo, establecimiento de pausas } \\
\text { y uso de EPP. }\end{array}$ \\
\hline
\end{tabular}

Fund. Prev. RL: Fundación para la prevención de riesgos laborales. Fuente: elaboración propia. 


\section{Conclusiones}

Si bien existen muchas empresas en el sector que cuentan con sus propios procesos, fue posible generalizar $6 \mathrm{de}$ estos para el estudio, y la variabilidad osciló entre 1 y dos procesos.

Se logró consolidar las actividades aisladas en un único plan de acción, bajo la conceptualización teórica de cada aspecto ergonómico, tanto para macro como para micro ergonomía, pasos generales para evaluar, caso aplicado en una empresa asociada a Acicam, y recomendaciones particulares para ser replicables en el sector.

Aunque se sugiere que las empresas adopten una distribución en forma de "U" o en línea, dependiendo del número de entradas y salidas que tengan, se encontró que no es posible generalizar el tipo de distribución de planta para las empresas. A diferencia del diseño organizacional y las capacitaciones que sí son posibles su generalización.

Se logró un rediseño que logra disminuir la carga física biomecánica sobre el puesto del trabajo y la tarea bajo la metodología design thinking, donde se permitieron propuestas innovadoras y centradas en el usuario

\section{Recomendaciones}

Se sugiere proponer diferentes estudios que se centren en el diseño de la herramienta y que atiendan a los problemas de posturas y movimientos repetitivos en el sector.

Se recomienda a las empresas del sector que si quieren hacer alguna intervención de los planes de acción deben tener presente el plazo en el cual se pretende intervenir.

\section{Agradecimientos}

Un especial agradecimiento a la Asociación Colombia de Industriales del Calzado, el Cuero y sus Manufacturas Acicam por su apoyo en la realización del estudio

\section{Referencias}

[1] OIT. Organización Internacional del trabajo. Sectores e Industria: textiles; vestido; cuero; calzado. 2016.

[2] L.B. Guimarães, J.L.D. Ribeiro, J.S. Renner, \& P.A.B. Oliveira, "Worker evaluation of a macroergonomic intervention in a Brazilian footwear company", Applied Ergonomics, vol 45 no 4, pp 923935, Jul. 2014.
[3] Instituto de Biomecánica de Valencia y Asepeyo, "Estudio ergonómico en puestos de trabajo del sector del calzado”, pp 1-65, 2010.

[4] S. Costa, S. Monteiro, P. Carneiro, A. Colim, N. Costa, I. Louderiro, "Ergonomic analysis of the shaping post in the injection sector of a footwear industry" en Occupaional Safety and Hygiene III. Guimaraes, Portugal. Taylor \& Francis, pp 227- 230, 2015.

[5] M. Baxley \& A. Kuhens, "Ergonomic Risk Exposure: Assessment of Safety Shoe Workers", Murray State University: Explorations, 2, pp 1-15, 2013.

[6] FASECOLDA. Federación de Aseguradores Colombianos. Prevención de la enfermedad laboral en Colombia, Colombia, 2016. Disponible en: http://www.fasecolda.com/index.php/sala-deprensa/noticias/2016/sector-febrero-16-2016/

[7] Ministerio de Comercio Industria y Turismo, "Programa de Transformación Productiva. Estructura Productiva", Colombia. 2013. Disponible en: http://ptp.amagi4all.com/informacion-

estadistica/cuero/cuero-estructura

[8] P. Aragón, \& K. Ordoñez, "Caracterización de los factores de riesgos ergonómicos por carga física biomecánica y condiciones de trabajo del subsector de calzado de empresas pertenecientes a ACICAM seccional Valle del Cauca”. tesis de pregrado. Dpto. Ing. Civil e Industrial. Pontificia Universidad Javeriana, Cali, Colombia, 2017.

[9] G. Morel, R. Amalberti, \& C. Chauvin, "How good micro/macro ergonomics may improve resilience, but not necessarily safety". Safety Science, Vol 24, no 2, pp. 285-294, 2009.

[10] R. Sampieri, C. Fernandez \& P. Baptista, "Metodología de la investigación". 6ta Ed, México, D.F., México, McGraw Hill, pp. 2-7, 2014.

[11] R.Curedale, "Design thinking: process and methods manual”. Topanga, United States: Design Community College Inc., 2013.

[12] T. Yang, C.T Su, \& Y.R. Hsu, "Systematic layout planning: a study on semiconductor wafer fabrication facilities". Int J Oper Prod Man, vol 20, no 11, pp 13591371, 2000.

[13] R. Daft, "Teoría y diseño organizacional". 10ma Ed, Santa Fe, México, Cengage learning, 2011. 